\title{
What to Show? Automatic Stream Selection Among Multiple Sensors
}

\author{
Rémi Emonet $^{1}$, E. Oberzaucher ${ }^{2}$, J.-M. Odobez ${ }^{3}$ \\ ${ }^{1}$ Laboratoire Hubert Curien UMR CNRS 5516, Université Jean Monnet, St-Etienne France \\ ${ }^{2}$ Dpt of Anthropology, University of Vienna, Austria \\ ${ }^{3}$ Idiap Research Institute, 1920 Martigny, Switzerland \\ remi.emonet@univ-st-etienne.fr, elisabeth.oberzaucher@univie.ac.at,odobez@idiap.ch
}

Keywords: stream selection, camera network, probabilistic models, temporal topic models

\begin{abstract}
The installation of surveillance networks has been growing exponentially in the last decade. In practice, videos from large surveillance networks are almost never watched, and it is frequent to see surveillance video wall monitors showing empty scenes. There is thus a need to design methods to continuously select streams to be shown to human operators. This paper addresses this issue and make three main contributions: it introduces and investigates, for the first time in the literature, the live stream selection task; based on the theory of social attention, it formalizes a way of obtaining some ground truth for the task and hence a way of evaluating stream selection algorithms; and finally, it proposes a two-step approach to solve this task and compares different approaches for interestingness rating using our framework. Experiments conducted on 9 cameras from a metro station and 5 hours of data randomly selected over one week show that, while complex unsupervised activity modeling algorithms achieve good performance, simpler approaches based on amount of motion perform almost as well for this type of indoor setting.
\end{abstract}

\section{Introduction and Previous Work}

There is an ever increasing number of sensors deployed in the real world, being it in large scale sensor networks or closed-circuit television (CCTV) networks. Exploitation of these cameras fulfill several needs: safety, when the physical integrity of people in a given environment need to be ensured (e.g., when people go in or out a metro train, or by detecting events that could lead to accidents in traffic infrastructures); security and protection of equipment through the detection of anti-social behaviors and vandalism; and efficiency, e.g., through the identification of typical flux and detection of congestions that can be communicated appropriately to users.

However, most of the time, surveillance network videos are never watched. For instance, in our case, 28 monitors are used in the control room to supervise more than 800 cameras. The probability to watch the right streams at the right time is therefore very limited. Moreover, the operator vigilance also appears to be a core criterion that strongly influences CCTV effectiveness, especially when a constant human monitoring is required. As vigilance studies confirm, operators who spend hours screen gazing at static scenes tend to become bored and less efficient, and are then likely to miss low-frequency events, such as a person falling or jumping over a turnstile.

Thus, along with top-down user-based protocols for visualizing and browsing the video networks, and the detection of predefined event, there is a need for bottom-up automatic and content-based selection systems that provide surveillance operators with the most salient and informative data streams within an adaptive real-time process. To address this issue, unusual event detection (Zhong et al., 2004; Boiman and Irani, 2007; Hua et al., 2009) and abnormality rating algorithms (Zelniker et al., 2008; Varadarajan and Odobez, 2009; Emonet et al., 2011) have been proposed in the past. Although these algorithms are obviously related to this paper, none of them has addressed the stream selection task as we propose in this paper.

Commercial systems tends to use the amount of motion and person tracking in order to select cameras with a high level of activity, in this paper we try to investigate how relevant this choice is. Recent related work also includes work that both model the content of the videos and monitor the operator with a camera such as in (Atrey et al., 2011). We consider a setup where observing the operator is not an option and where only the content of the videos is used to 
select the stream to display.

In this article, we explore the stream selection issue. First, in Section 2, we formalize the task. Then, in Section 3, we propose an approach for collecting ground truth for the task, based on human estimation of interestingness done by 100 persons on videos coming from 9 cameras and spread over one week. Section 4 describes the approaches used to solve this task together with the performance measure we propose to evaluate these approaches. The experimental results are detailed in Section 5. Finally, our conclusions and future work is presented in Section 6.

\section{Defining the Stream Selection Task}

The ultimate goal of stream selection algorithms is to allow the automatic pre-selection of some audio or video streams to present to a human operator for further screening. More formally, given a set of $N$ streams, the system must continuously select the $M$ ones that are the most meaningful to show to an operator. Defining what relevance criterion has to be used is an open problem and in the following sections, we use human attention as a cue. Also, even if not considered in our evaluations, the selection must be consistent in time, for example it should avoid screen flickering and avoid showing continuously the same views (unless it is sure these are very relevant).

Figure 1 illustrates the context and the task. In Figure 1a), an operator looking at a video wall is depicted. In Figure 1b) the stream selection task is shown, with the $N$ input streams and the $M$ streams selected by the system and to be shown to the operator.

\section{Social Attention and Ground Truth}

The ground truth dataset eventually used for the evaluation of the stream selection task is based on an experiment on social attention, described in Section 3.1. We follow the idea that human attention can act as a guide for the selection of interesting sensors.

\subsection{Button clicking experiments}

We carried out an experiment where we simultaneously showed multiple movies to our subjects, and they had to press a button corresponding to the screen that showed something interesting. For this purpose, we created a set of 2-minute long movie chunks. We

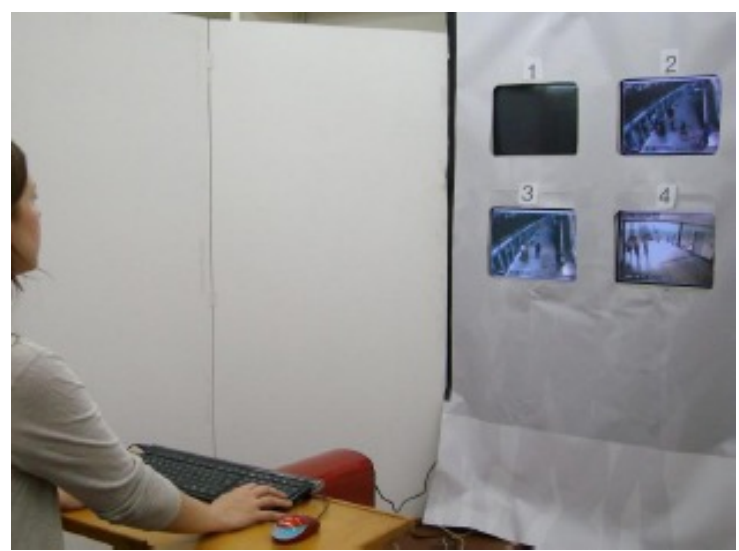

Figure 2: The setup for the Virtual Operator experiment on social attention. The subject is exposed to 4 synchronized videos. Whenever something interesting is happening on one of the screen, the user have to press the button corresponding to the screen. Every two minutes, the videos are changed; and b) an illustration of the window-based and Gaussian-smoothing-based filtering for button clicks.

considered 9 cameras from a camera network deployed in a public transportation system. We randomly picked 150 starting times across a week period, and for each starting time, we created 2-minute chunks for all 9 camera views starting at these times. These $150 \times 9$ movies are the basis for the attention study.

An environment called the "Virtual Operator" have been used for data collection. Figure 2 shows a picture of the virtual operator setup. The virtual operator simultaneously shows four synchronized video streams on a 2 by 2 grid of screens. Each screen shows a shot from a different surveillance camera. Every two minutes, a new set of movies is randomly brought up to the screens.

Whenever the subject sees something interesting on one of the screens, he or she has to press a button indicating the respective monitor. The participants had to fill out some questionnaires prior to and after the experiment. In total 52 male and 51 female subjects (mean age 23.78) participated in the study. Each 2 minute chunk was watched between 14 and 18 times.

First, we see that attention is clearly not randomly distributed over the movies. The random probability of two subjects hitting the same button at the same time (same frame, at 5 frames per second) is 0.0016 . By analyzing the distribution of the button clicks, we observe that clicks tend to cluster around some instants and views of interest.

Overall, we can retained the following observations: a) the attention is not randomly distributed, b) 
a)

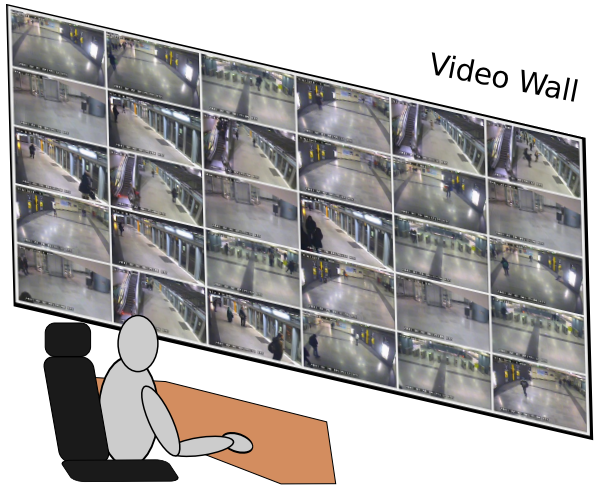

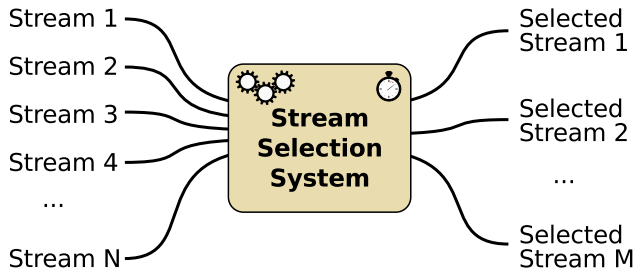

b)

Figure 1: a) An operator in front of a video wall. b) Stream selection task: at any time instant, select the $M$ streams, among $N$, that are the most meaningful to show to a human operator.

there are certain scene properties that suggest attention, and c) there is some degree of random clicks. This experiment suggests that, once cleaned, the button clicks can be a good indicator of the video stream interestingness. We thus use these interestingness clicks for the creation of a ground truth, making the assumption that the interestingness clicks are related to which stream should be selected in the task of stream selection.

\subsection{Derived ground truth}

From the button clicks, we derive a "annotation database" that we will use for the evaluation of stream selection algorithms. Overall, the annotation database can be seen as a list of annotated instants where people marked a single view. Each instant has a timestamp, the ordered list (set) of the views shown and the index of the view that was marked by the subject. More formally we can define the annotation database as:

$$
A=\left\{A_{i}\right\}_{i=1 . . n}=\left\{\left(t_{i}, V_{i}, u_{i}\right)\right\}_{i=1 . . n}
$$

where $n$ is the number of annotations, $A_{i}$ is the $i^{t h}$ annotation in the list, $t_{i}$ is its timestamp, $V_{i}$ is the list of shown views and $u_{i}$ is the index of the view marked as interesting $\left(V_{i}\left(u_{i}\right)\right.$ thus being the selected view). In the raw button click dataset presented before, there are 9 possible views (i.e., each element of $V_{i}$ can take 9 different values) and $n$ is almost 10000 and realized by around 100 subjects. The timestamp is a pointer to any instant (a frame) within one of the 150 slots (each, long of 2 minutes) that were extracted over one full week. Each annotation provide information about the selected view versus the 3 others but does not provide information between these 3 other views.

There is a actually a mismatch between the button clicks and the goal of the stream selection system: the complete system should be able to continuously select which stream(s) to show, while the button clicks are sparse. For the evaluation measure presented in Section 4.1, we thus make the assumption that only the instant that have been clicked by some users are meaningful for the evaluation. Said the other way around: on the periods where there are no clicks, the selection algorithms can produce any results without being penalized.

\subsection{Robust ground truth}

We observed some user clicks are random. Also, as some subjects might be quicker to respond than others, it is problematic to instantaneously link the button clicks to the respective scene in the video. Apart from considering the raw button clicks we also considered a smoothed version of the "annotations".

We run a Gaussian smoothing function over the click results, generating a continuous index of interestingness (IoI). The IoI is taking into account multiple button presses within a small time span accounting for the temporal uncertainty of the raw button clicks. A threshold on the IoI value is used to decide whether an annotation should be kept. The threshold can be tuned to control the robustness of the resulting dataset. The provided results are obtained with around 10000 annotation for the raw case and around 2000 for the smoothed one.

\section{Experimental setup}

The goal of a stream selection system is to select the $M$ among $N$ streams to display to an operator. Almost equivalently, we can consider that the system task is to rank the streams by interestingness and the first ones are displayed. We thus suppose 
that the output of an algorithm algo at any time instant $t^{\text {eval }}$ and for a set of views $V^{\text {eval }}$ is a ranking of the camera views (or their indices). For instance, $\operatorname{algo}\left(t^{\text {eval }}, V^{\text {eval }}\right)=(3,1,2,4)$ means that the third streams is the most interesting to show, then the first stream, then second and fourth. For performance measurement, we make the assumption that the instants where stream selection matters are only the ones which have been clicked by the human subjects.

\subsection{Performance measure}

At each marked instants, the ideal selection algorithm would rank first the stream that was marked by the human subject. A less effective algorithm would rank this stream second, and so on, the worse in our case being to rank it 4 (as there are 4 views shown at any time). To analyze the performance of an algorithm, we can thus look at the distribution of the rank produced by the algorithm for the clicked instant. More precisely, we compute the proportion of times the algorithm ranked the mark stream first, second, third and fourth:

$$
\begin{aligned}
& \forall r \in 1 . .4 \\
& \begin{aligned}
p^{\text {algo }}(r) & =\frac{1}{n} \times \operatorname{Card}\left\{i \mid \operatorname{algo}\left(t_{i}, V_{i}\right)(r)=u_{i}\right\} \\
& =\frac{1}{n} \times \text { num. clicked } u_{i} \text { ranked } r \text { by algo }
\end{aligned}
\end{aligned}
$$

This distribution $\left(p^{\text {algo }}(r)\right)$ in itself is a complete description of the performance of the algorithm. From this distribution, we could extract more synthetic numerical performance measures by giving different weights to the different ranking errors. For example, if we are interested in the percentage of time the algorithm performs in perfect accordance with the annotations, we can just take the value of $p^{\text {algo }}(1)$. Average precision is algo a common measure and corresponds to $\sum_{r=1}^{4} \frac{1}{r} p^{\text {algo }}(r)$.

\subsection{Modular architecture for selection}

The stream selection algorithms are responsible for providing a ranking or a selection of the cameras to show to the operator. Most algorithms are providing an interestingness rating at each instant for a camera. If we want to be able to integrate information across cameras and to mix different algorithms in a system (some can be more effective on some cameras), we have to consider a kind of normalization of the measures across cameras. The overall stream ranking system follows the architecture shown in the Figure 3:
- each video stream is processed independently by the interestingness/abnormality rating algorithm;

- a possible normalization step is performed in order to provide comparable abnormality measures across views and algorithms. Normalization parameters are typically learnt without supervision using statistics on the abnormality measure on some (unlabeled) learning videos.

- the normalized abnormality scores are used as the inputs of a ranking algorithm whose goal is to produce the final ranking of the camera views. The simplest ranking algorithm (that we have used in the reported experiments) just ranks the views according to their normalized abnormality score. Temporal integration (not done here), which ensures stability in the views shown to the operator, would also take place in the final ranking algorithm.

In the experiments, we consider a single stream ranking algorithm: it simply sorts the streams based on their normalized interestingness measure. For the stream interestingness rating algorithms and for the normalization algorithms, we considered different algorithms detailed in the following subsections.

\subsection{Considered interestingness rating algorithms}

We consider multiple algorithms: two simple baseline algorithms, a method from the state of the art (Emonet et al., 2011) with variations, and a mixture of methods.

$\underline{\text { Random - we just simulate a random interestingness }}$ score at any instant. This pseudo-algorithm is used only to illustrate the random factor inherent to the task. This random baseline can also be seen as a random ranking of the 4 views of interest. Overall, it is equivalent to ranking all the views in a uniform random manner and thus having $25 \%$ of probability for any of the 4 ranks.

Motion - we use the amount of motion as an interestingness measure given that studies on human attention have shown correlation between motion and attention. The abnormality score for each view is simply the amount of detected motion (proportion of image pixels where a motion could be robustly detected) using a Lucas Kanade method. With no normalization, this score will tend to display the camera with absolutely more motion. With some per-view normalization, the score will favor cameras with more motion than the view average.

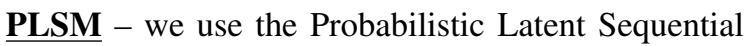




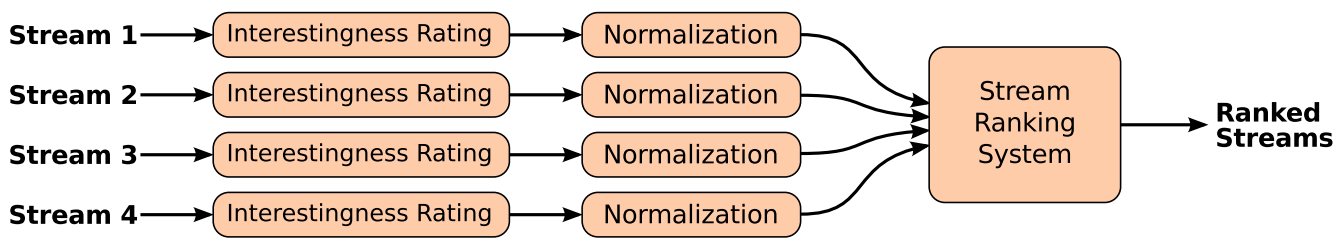

Figure 3: Architecture for the stream selection. Each stream is processed independently: a first algorithm is extracting an interestingness measure and this measure is then normalized. The normalized measures are used by a simple algorithm that ranks the streams based on their measures.

Motifs (PLSM) that has been used in (Emonet et al., 2011) to extract an abnormality measure from video. The principle of PLSM is to learn, in an unsupervised way, the temporal motifs that are recurrent in a temporal document. Applied to a video with localized motion features, the algorithm learns the typical activities, which are often typical trajectories of objects within the scene. The authors of (Emonet et al., 2011) suppose that the recurrent motifs represent the usual, normal activities and use this supposition to derive an abnormality score. We use the abnormality score as an interestingness measure. PLSM has been run, on each view, on 2 hours of video data with the following parameters: 75 topic at low-level, 20 motifs of 15 seconds each.

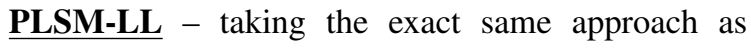
PLSM, we also experimented with using the negative log-likelihood of the observations after the fitting of the PLSM model. The same parameters as for the PLSM measure have been used.

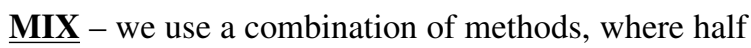
of the camera views are rated with "Motion" while the other half are rated with "PLSM".

\subsection{Considered Normalization Methods}

The interestingness rating produced by different algorithms and/or for different camera views might vary notably in their scale. To be able to merge the views in a meaningful manner, we need to re-scale them to a common range of values. Many ways of normalizing the scores can be imagined and implemented.

In our case we only consider automatic ways of re-normalizing the abnormality scores. All the normalization parameters are set using a learning datasets of 2 hours (without any supervision or annotation). The abnormality score is first extracted on this data (at each time instant) and then the resulting abnormality time series is analyzed to produce the normalization parameters. We considered two kind of normalization methods.

nonorm $(\varnothing)$ - we just simply don't normalize the ab- normality measure. Given the discrepancy between different rating algorithms, this method is limited to the cases where only a single type of interestingness algorithm is used. Still, it makes sense to try this normalization (actually non-normalization). For example, the "motion" measure without normalization will actually favor the selection of the view with the absolute most motion. From the experiments, it can also be meaningful to not normalize the interestingness measure obtained from PLSM as it is also related to motion.

percent99 (p99) - we explored a normalization based on the $99^{\text {th }}$ percentile. Using the learning set, we linearly re-normalize the abnormality using a scaling factor $p_{99}$ such that the observed interestingness score falls below a fixed threshold (e.g., 1) $99 \%$ of the time. The scaling factor is then used to proportionally rescale the abnormality, i.e, norm.score $=\frac{\text { score-minscore }}{p_{99}-\text { minscore }}$. This normalization can be seen as a robust equivalent of re-normalizing using the maximum observed value.

percent95 (p95) - as with "percent99", we experimented with a normalization based on other percentiles, only $95 \%$ being shown (see results). We also experimented with other percentiles: for high percentiles, results are comparable to the $99^{t h}$ and $95^{\text {th }}$ one, while smaller percentiles lead to lower results.

\section{Results}

We provide results using the two versions of the button clicking annotations. First with the raw annotations (about 10000 clicks), then with the Gaussiansmoothing approach (about 2000 clicks).

\subsection{Results using the raw ground truth}

We first evaluate the algorithms introduced previously on the complete ground truth. As mentioned in Section 3.3, this ground truth is actually noisy as the human subject do not agree exactly on the interesting 
streams. We evaluate the different algorithms together with the different normalization methods. Our algorithms are compared against a theoretical random algorithm that would rank the views randomly (no normalization is meaningful).

As for example in Figure 4, the result figures show the rank distributions for various interestingness algorithm, grouped by normalization method. In a given graph, there is a line per considered method. For a given method, the colored bars show the proportion of clicked views ranked $1^{s t}, 2^{\text {nd }}, 3^{\text {rd }}$ and, $4^{\text {th }}$ by the algorithms. The red bar corresponds to $p^{\text {algo }}(1)$ and so on. More weight on the first (leftmost) ranks is better. We see for example that random (which is repeated on each graph) has a probability of 0.25 for each of the ranks. Methods that will be above 0.25 for the first rank, can be considered as better than random.

The normalization based on percentiles below 95\% give systematically lower performance than the one with $95 \%$. To improve readability, we thus omit these in the shown graphs. The results show that the consider algorithms, both motion based and PLSM based, perform better than the random guess. Despite the fact that the raw annotations have been found relatively noisy, the gain over random algorithms is important.

The results also show that the simple motionbased criterion is almost as good as the more elaborated PLSM algorithm. The main observation is that in this metro setting, human social attention, encoded in the form of our annotations, is very well linked to the amount of motion present in the video. Both kind of abnormality measures derived from PLSM seem also to be following the behavior of "Motion". However, in other contexts like traffic videos, where motion is present in the form or more regular patterns, the capacity of algorithms such as PLSM at filtering out normal activities would be beneficial to detect anomalies as compared to relying on motion only.

In this setup, we observe that normalization has a small negative on the results. The interpretation can be that human attention is directed toward the absolute amount of motion and not a motion amount relative to the normal amount. Actually, noise in the annotations is also part of the reason as shown in the following section.

\subsection{Results on filtered dataset using the Gaussian smoother}

We also evaluate the performance of the different algorithms on the ground truth after smoothing by a Gaussian kernel, as explained in Section 3.3. We varied the threshold on the smoothed index of interest so as to keep around 2000 evaluation points. Figure 5 shows results with the corresponding results.

Globally, the methods exhibit better accuracy on the cleaned ground truth. We also observe a more marked effect of method mixing: results are notably lower than with each individual methods. When mixing methods, normalization also helps. However, as observed in the raw case, normalization degrades the results for individual methods. This again means that the attention is directed to absolute motion.

Overall, the results with the smooth annotations consolidate and complete what has been observed with the raw annotations:

- non-normalized version are preferable for both the motion-based measure and the PLSM-based one,

- when mixing methods of different scales, as expected, results degrades and the normalization helps,

- motion-based and PLSM-based selection both significantly outperform the random guess algorithm especially on cleaned ground truth,

- on the raw ground truth, the motion-based selection works best,

- motion-based methods and PLSM-based methods perform comparably,

- we reach good ranking accuracy of up to 0.6 meaning that $60 \%$ of the times the stream clicked by the human subject is ranked first among four streams; $85 \%$ of the times, the clicked stream is among the two firsts in the rank provided by the algorithm.

\section{Conclusions and future work}

We have introduced the stream selection task and proposed an evaluation protocol for it. The proposed evaluation is based on a social attention experiment where human subjects were shown four video streams at once and were asked to mark a video at any time they spotted something interesting in it. We tested various stream selection algorithms to rate the interestingness of the streams at any instant. The video instants marked by the subjects are used to evaluate how well the marked video has been ranked by a selection algorithm.

Our evaluations have shown that, considering all the raw annotations produced by the human subjects, all test approaches were better than random but mostly equivalent to a simple criterion based on the amount of motion. By filtering the annotations (removing the isolated ones) we obtained a more trustful 


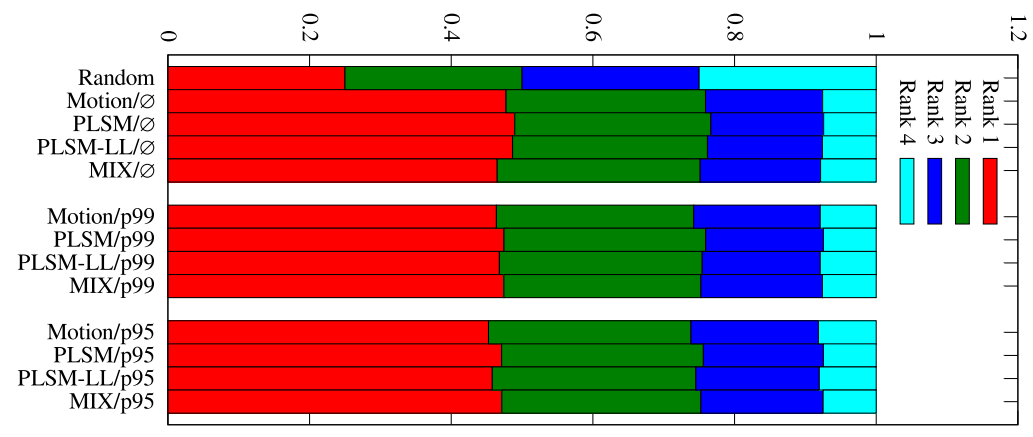

Figure 4: Ranking results on the raw ground truth (9980 evaluation instants) coming directly from the button clicking experiment. For each method (row), the proportion of each rank position is given for the considered annotation points. More weight on the first (leftmost) ranks is better.

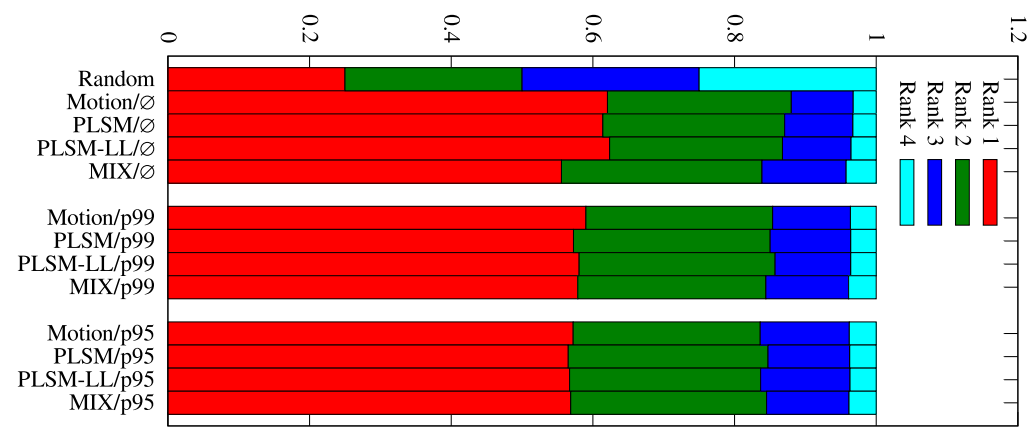

Figure 5: Ranking results on the smoothed ground truth obtained by removing annotations with unsufficient supporting evidence (total, 2003 evaluation instants).

set of annotations. On such more robust evaluation dataset, it has been shown that mixing interestingness measures of different scale actually poses problem and that the proposed normalization method helps in this case.

Overall, the results are encouraging and more interestingness rating algorithms need to be tested and evaluated in this framework. More advanced normalization methods needs also to be explored: we can expect to find a normalization procedure that allows to mix various algorithms without degrading their individual accuracy. We plan to explore a normalization based on robustly aligning every percentile of all interestingness measures. A possible per-view bias (e.g., a particular view being almost never interesting) could also be integrated in the normalization process if we accept some slight human supervision. Collecting more ground truth, especially with real operators which have been knowing the camera setup for months, and making it available for research purpose is also in our future work direction.

\section{Acknowledgement}

This work was funded by the European Union FP7 project VANAHEIM (248907) under the 7th framework program. 


\section{REFERENCES}

Atrey, P. K., El Saddik, A., and Kankanhalli, M. S. (2011). Effective multimedia surveillance using a humancentric approach. Multimedia Tools and Applications, 51(2):697-721.

Boiman, O. and Irani, M. (2007). Detecting irregularities in images and in video. International Journal of Computer Vision, 74(1):17-31.

Emonet, R., Varadarajan, J., and Odobez, J.-M. (2011). Multi-camera Open Space Human Activity Discovery for Anomaly Detection. In 8th IEEE Int. Conf. on Advanced Video and Signal-Based Surveillancei (AVSS), page 6 .

Hua, D., Zhang, X., Yin, J., Zheng, V. W., and Yang, Q. (2009). Abnormal activity recognition based on hdphmm models. In Int. Joint Conference in Artificial Intelligence.

Varadarajan, J. and Odobez, J. (2009). Topic models for scene analysis and abnormality detection. In ICCV12th International Workshop on Visual Surveillance.

Zelniker, E., Gong, S., and Xiang, T. (2008). Global abnormal behaviour detection using a network of cctv cameras. In IEEE International Workshop on Visual Surveillance.

Zhong, H., Shi, J., and Visontai, M. (2004). Detecting unusual activity in video. In IEEE Conference on Computer Vision and Pattern Recognition, volume 2, pages 819-826, Washington, DC. 